\title{
DETERMINAÇÃo DE ASCAREL EM ÓLEO ISOLANTE DE TRANSFORMADORES
}

\author{
Izoldir Antonello, Eduardo Humeres, Ivan Gonçalves de Souza e Nito Angelo Debacher* \\ Departamento de Química, Universidade Federal de Santa Catarina, CP 476, 88040-900 Florianópolis - SC, Brasil \\ Alexandre Rios Martins \\ Centrais Elétricas de Santa Catarina, BR 101 km 215, 88132-300 Palhoça - SC, Brasil
}

Recebido em 20/10/05; aceito em 14/6/06; publicado na web em 19/1/07

\begin{abstract}
ASCAREL DETERMINATION IN INSULATING OIL OF TRANSFORMERS. The contamination level of silicon oil used as insulation liquid in high-voltage transformers by ascarel (PCBs) is above those permitted by the Brazilian law. Thus new techniques able to detect ascarel, with low operational costs, are very attractive. The present work proposes an analysis of the contamination levels of silicon oil using the following techniques: naphthalene anion radical reaction for ascarel dechlorination; and potentiometry with an ion-selective electrode for chloride ion determination. The data obtained with the proposed methodology agree well with those from the official methodology, (method IEC 61619).
\end{abstract}

Keywords: ascarel; PCBs; dechlorination.

\section{INTRODUÇÃO}

A rede de transmissão de energia elétrica usa transformadores de grande porte com capacidade elevada operando ininterruptamente durante anos. A durabilidade e o funcionamento adequado destes aparelhos depende de um eficiente sistema de isolamento e refrigeração, os quais são realizados por fluidos com propriedades tais como alta constante dielétrica e elevada estabilidade térmica. Até o início da década de 80 os fluidos usados para esta finalidade eram compostos organoclorados conhecidos por PCBs ("PolyChlorinated Biphenyls"), ou bifenilas policloradas. Os PCBs foram produzidos por várias empresas internacionais com diferentes nomes, tais como: ascarel ou askarel, fenoclor, aroclor, entre outros, sendo comercializados no Brasil como ascarel. A nomenclatura para definir sua composição possui características próprias com 4 dígitos, como por ex., aroclor 1254. Os dois primeiros algarismos representam o tipo molecular (12 átomos de carbono) e os dois últimos a porcentagem de cloro na composição do produto comercial. Assim, o produto aroclor 1254 é uma mistura de bifenilas policloradas contendo $54 \%$ de cloro $^{1-4}$.

$\mathrm{O}$ ascarel é comercializado como sendo uma mistura composta de $75 \%$ da espécie aroclor 1254 e $25 \%$ de triclorobenzeno. Outros tipos também são conhecidos como ascarel do tipo A, $60 \%$ da espécie 1260 e $40 \%$ de triclorobenzeno; e ascarel do tipo B, $70 \%$ da espécie 1254 e $30 \%$ de triclorobenzeno ${ }^{4}$. Devido à sua alta toxicidade e risco ao meio ambiente o uso dos PCBs foi proibido no Brasil a partir de 1981, através da Portaria Interministerial nº19, de 2 de Janeiro de $1981^{1-5}$.

A substituição do ascarel na indústria de transformadores foi feita preferencialmente pelo líquido de silicone ou "óleo silicone". No entanto, no processo de substituição, em alguns casos, ocorreu a contaminação do óleo silicone por ascarel devido à falta de critérios técnicos. Desta forma, muitos dos grandes transformadores ainda em operação no Brasil estão contaminados com ascarel em níveis acima dos permitidos pela legislação vigente ${ }^{6}$.

Atualmente para o setor de transmissão de energia elétrica ${ }^{7}$, são considerados equipamentos elétricos isentos de ascarel aqueles cujo

*e-mail: debacher@qmc.ufsc.br óleo mineral isolante contenha teores inferiores a $3,0 \mu \mathrm{g} \mathrm{mL}^{-1}$, não contaminados com valores inferiores a $50 \mu \mathrm{g} \mathrm{mL}^{-1} \mathrm{e}$ contaminados entre $50-500 \mu \mathrm{g} \mathrm{mL}^{-1}$.

O método oficial usado hoje para detecção e quantificação do ascarel é a cromatografia gasosa empregando colunas capilares (método IEC 61619$)^{8}$. Este método exige laboratórios especializados e técnicos qualificados, onerando o processo. Além disso, há a necessidade do transporte das amostras até o laboratório causando demora na emissão dos resultados. Desta forma, novos procedimentos capazes de detectar o ascarel in loco, apresentando baixos custos operacionais e rapidez na emissão de resultados, caracterizam-se como atrativos interessantes para uma técnica de controle deste poluente. $\mathrm{O}$ presente trabalho propõe um procedimento semi-quantitativo de detecção de ascarel empregando a desalogenação (remoção do cloro da molécula), via reação ânion radical naftaleno $(\mathrm{ARN})^{9-12}$ e determinação do íon cloreto por potenciometria usando eletrodo seletivo a cloreto ${ }^{13}$.

\section{MATERIAIS E MÉTODOS}

Para os procedimentos de síntese e caracterização, todos os reagentes e solventes empregados foram de grau analítico. A secagem do tetraidrofurano (THF) foi realizada empregando procedimento descrito na literatura ${ }^{14} \mathrm{e}$ o monitoramento do teor de água, empregandose o método Karl-Fischer. Padrões de $\mathrm{NaCl}(3,55$ - 35,5 - $355 \mu \mathrm{g} \mathrm{mL}$ $\left.{ }^{1}\right)$ foram empregados para os procedimentos potenciométricos. As análises cromatográficas foram realizadas empregando-se um cromatógrafo gasoso modificado CG-37 (column phase $\mathrm{AT}^{\mathrm{TM}}-5,60$ $\mathrm{m}, 0,25 \mathrm{~mm}, 1,0 \mu \mathrm{m}$, ECD). Utilizou-se nos experimentos um potenciômetro Orion modelo 920A com eletrodo seletivo para cloreto, Orion Chloride Plus, 9617. As amostras para análise foram obtidas junto a Centrais Elétricas de Santa Catarina - CELESC.

\section{PROCEDIMENTOS}

O procedimento experimental de análises adotado levou em consideração a possibilidade do ensaio ser realizado no campo de forma simples, sem necessidade de pessoal especialmente treinado. O procedimento foi dividido em três etapas: desalogenação do 
ascarel contido na amostra; extração dos íons cloreto da fase orgânica; e, detecção potenciométrica dos íons cloreto livres.

Para os testes experimentais foram usadas amostras padrão de óleo mineral isolante de transformador contaminado por ascarel entre $0-200 \mu \mathrm{g} \mathrm{mL}^{-1}$. A reação de desalogenação do ascarel em amostras de óleo isolante foi feita via ânion radical naftaleno (ou naftaleto de sódio) (ARN), preparado conforme descrito a seguir ${ }^{15,16}$. Em um balão de três bocas com sistema de refluxo e agitação magnética foram adicionados $10 \mathrm{~g}$ de naftaleno $(0,078 \mathrm{~mol}), 1,8 \mathrm{~g}$ de sódio metálico $(0,078 \mathrm{~mol})$ e $60 \mathrm{~mL}$ de THF $(0,740 \mathrm{~mol})$. A formação do produto foi acompanhada durante $2 \mathrm{~h}$ por espectrofotometria na região do visível pelo aparecimento da banda em $550 \mathrm{~nm}$ correspondente à cor verde do ARN. O produto foi armazenado a $-10^{\circ} \mathrm{C}$, na ausência de luz e umidade, para posterior utilização.

A reação de desalogenação foi realizada em um funil de separação, usando diferentes quantidades de amostra (25,0 a 50,0 mL), adicionando-se lentamente de 2,0 a 5,0 mL do ARN sob agitação durante 10 a 15 min. A quantidade de ARN adicionado na amostra e o tempo de agitação foram controlados visualmente, observando-se a persistência da coloração verde do ARN na solução. A persistência da cor verde indica excesso de ARN e o término da reação, ou seja, a desalogenação completa do ascarel na amostra ${ }^{16}$.

O ânion radical naftaleno ARN é estável a baixas temperaturas $\left(-10{ }^{\circ} \mathrm{C}\right)$ e em ausência de luz, no entanto, é extremamente sensível à umidade do ar e qualquer vestígio de água no meio reacional produzirá íons $\mathrm{OH}^{-}$por degradação. Desta forma, a extração dos íons cloreto do meio reacional para a fase aquosa foi realizada em três extrações de $10 \mathrm{~mL}$, sendo a primeira em meio ácido $(10 \mathrm{~mL}$ de $\mathrm{HNO}_{3}$ 0,1 mol L-1), para auxiliar a separação das fases e a neutralização dos íons $\mathrm{OH}^{-}$, os quais interferem nas leituras potenciométricas.

Para determinação do íon cloreto, o ajuste da força iônica das soluções foi realizado pela adição de $10 \mathrm{~mL}$ de tampão acetato 0,5 mol L-1 $(\mathrm{pH} 4,5)$ para um volume total de $50 \mathrm{~mL}$. As medidas potenciométricas foram realizadas com eletrodo seletivo a cloreto previamente calibrado com padrões de $\mathrm{NaCl}(3,55$ - 35,5 - 355 $\left.\mu \mathrm{g} \mathrm{mL}^{-1}\right)^{17}$. Deste modo, o teor de cloreto na amostra era fornecido em $\mu \mathrm{g} \mathrm{mL}^{-1}$, diretamente no visor do potenciômetro ${ }^{13}$.

Para aferição do procedimento proposto, foram empregados padrões certificados com teores de ascarel determinados pelo método IEC $61619^{8}$, no laboratório da CELESC.

Em todo o procedimento experimental, a reação de desalogenação do ascarel na amostra de óleo isolante padronizada, a extração dos íons cloreto com água e sua determinação por potenciometria foram realizadas em duplicata. Para a amostra D, entretanto, foi realizado apenas um ensaio.

\section{RESULTADOS E DISCUSSÃO}

As reações de desalogenação de haletos de alquila com ânion radical naftaleno (ARN) são bem conhecidas e podem ser divididas em quatro sub-reações: redução, redução bimolecular, monoalquilação e dialquilação, tendo como produtos da alquilação o 1,2 e 1,4-dihidronaftalenos ${ }^{18}$. No entanto, as reações de desalogenação com haletos de arila com ARN não estão totalmente elucidadas, mas sabe-se que podem ocorrem através de redução, com bases fortes, já que não reagem com a maioria dos nucleófilos. O mecanismo sugerido ocorre via eliminação/adição com formação intermediária do benzino ${ }^{19}$. De acordo com Smith e Bubbar ${ }^{20}$, estas reações são caracterizadas por rapidez e eficiência, fornecendo resultados quantitativos mesmo à temperatura ambiente. A reação de ARN com hidrocarbonetos polihalogenados é igualmente descrita por Parker e Steichen ${ }^{21}$ e Brunelle ${ }^{22}$.

Os resultados mostram que a desalogenação do ascarel em amostras de óleo mineral via reação ARN é rápida, levando entre 10 a 15 min, e de fácil acompanhamento visual, em função da mudança de coloração do meio. O ARN possui coloração verde e a permanência da coloração no meio reacional indica excesso de $\mathrm{ARN}$, portanto término da reação. A quantidade de ARN empregado na reação varia de acordo com a concentração de ascarel na amostra e da quantidade de água no meio orgânico. Como o ARN é extremamente sensível à água no meio reacional produzindo íons $\mathrm{OH}^{-}$por degradação, todo o excesso de ARN é degradado, quando da adição de água, no processo de extração dos íons. Desta forma, é importante a neutralização da fase aquosa pois os íons $\mathrm{OH}^{-}$interferem nas leituras potenciométricas. Sendo que, na presença de quantidades iguais de íons $\mathrm{Cl}^{-}$e $\mathrm{OH}^{-}$, pode se obter erros de até $0,5 \%$ na determinação de cloreto. Outros íons, tais como $\mathrm{Br} ; \mathrm{I} ; \mathrm{CN}^{-} ; \mathrm{S}^{-2}$ e $\mathrm{NH}_{3}{ }^{13}$, também interferem na leitura.

O ajuste da força iônica do meio, requerida pelo método potenciométrico empregando eletrodos seletivos a íons, realizado através da adição de $10 \mathrm{~mL}$ de tampão acetato $0,5 \mathrm{~mol} \mathrm{~L}^{-1}(\mathrm{pH} 4,5)$, para um volume final de $50 \mathrm{~mL}$, mantém a força iônica igual a 0,1 . Este valor é aproximadamente 10 vezes maior que a concentração do padrão de cloreto $355 \mu \mathrm{g} \mathrm{mL} \mathrm{mL}^{-1}$.

A Tabela 1 apresenta os resultados comparativos da quantificação de ascarel em amostras de óleo isolante contaminado, empregando o procedimento proposto e a metodologia oficial (método IEC 61619) ${ }^{8}$.

O desvio padrão da $3^{\text {a }}$ coluna (Tabela 1 ) onde aparecem os valores da concentração de íons cloreto na amostra, determinados potenciométricamente, a média de duas medidas, exceto para a amostra D, onde foi realizado apenas um ensaio. As amostras A e B e E e F, respectivamente, possuem pelo método IEC 61619 o mesmo teor de ascarel, porém como são de procedências diferentes o teor de triclorobenzeno poderá não ser o mesmo. Assim, para efeitos comparativos foram consideradas em separado.

O procedimento proposto considera a desalogenação de todas as espécies organocloradas existentes na amostra de óleo isolante (ascarel e triclorobenzeno), sem especificar a porcentagem de átomos de cloro na amostra. Já a cromatografia gasosa distingue entre os tipos de aroclor (aroclor 1242 (42\% de cloro), 1254 (54\% de cloro), 1260 (60\% de cloro)) e exclui componentes que eluem até $15 \mathrm{~min}$, entre eles o triclorobenzeno e seus possíveis isômeros além de mono, di e tetra-clorobenzeno. Isto justifica a detecção de íons cloreto por potenciometria nas amostras $\mathrm{G}$ e H (Tabela 1, $3^{\mathrm{a}}$ coluna), supostamente isentas de ascarel, além de teores mais elevados de $\mathrm{Cl}^{-}$para as amostras A, B, E e F.

$\mathrm{Na}$ tentativa de expressar os valores em $\mu \mathrm{g} \mathrm{mL}^{-1}$ de ascarel (Tabela $1,7^{\text {a }}$ coluna) aplicamos a regra de três simples aos dados da $3^{\text {a }}$ coluna da tabela, da seguinte forma: para a amostra A, temos: $(21,9 \times 100 \% / 42 \%=52,1)$, o mesmo foi feito para a espécie aroclor 1254 e 1260. A média dos três valores obtidos (Tabela 1, amostra A) foi $(52,1+40,6+36,5) / 3=43,1 \pm 8,1$. O desvio padrão de $\pm 8,1$ neste caso indica apenas que por aproximação podemos considerar que o procedimento proposto é adequado para determinar faixas de contaminação de $\pm 50 \mu \mathrm{g} \mathrm{mL}^{-1}$, o que está em concordância com a norma técnica vigente, NBR 8371/946.

Para efeitos práticos podemos apenas multiplicar o resultado de íons cloreto por dois pois a média aproximada de cloro em peso na molécula, entre os três tipos de aroclor no ascarel (42, 54 e 60\%), corresponde a aproximadamente a 52\%. Desta forma, para obter o valor da porcentagem total $(100 \%)$ de ascarel na amostra, pode-se multiplicar por dois os valores de íons cloreto detectados potenciometricamente, (Tabela 1).

Os resultados obtidos demonstram que o procedimento proposto de desalogenação via ARN e a determinação de íons cloreto por potenciometria é adequado para indicar com segurança o grau de 
Tabela 1. Resultados comparativos de quantificação de ascarel em amostras de óleo isolante, empregando o procedimento proposto (potenciometria) e a metodologia oficial (método IEC 61619) ${ }^{8}$

\begin{tabular}{|c|c|c|c|c|c|c|c|}
\hline Amostras de $\mathrm{OMI}^{\mathrm{a}}$ & $\begin{array}{c}\text { Método } \\
\text { IEC } 61619\left(\mu \mathrm{g} \mathrm{mL} \mathrm{mL}^{-1}\right)^{\mathrm{a}}\end{array}$ & $\begin{array}{l}\text { Potenciometria } \\
\mathrm{Cl}^{-}\left(\mu \mathrm{g} \mathrm{mL}^{-1}\right)^{\mathrm{b}}\end{array}$ & $\begin{array}{c}1242 \\
\left.(\mu \mathrm{g} \mathrm{mL})^{-1}\right)^{\mathrm{c}}\end{array}$ & $\begin{array}{c}1254 \\
\left.(\mu \mathrm{g} \mathrm{mL})^{-1}\right)^{\mathrm{c}}\end{array}$ & $\begin{array}{c}1260 \\
\left.(\mu \mathrm{g} \mathrm{mL})^{-1}\right)^{c}\end{array}$ & 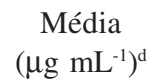 & $\begin{array}{l}\text { Resultado } \\
\text { Aceito }^{24}\end{array}$ \\
\hline A & 25,0 & $21,9 \pm 2,6$ & 52,1 & 40,6 & 36,5 & $43,1 \pm 8,1$ & $\operatorname{sim}$ \\
\hline B & 25,0 & $20,2 \pm 1,3$ & 48,1 & 37,4 & 33,7 & $39,7 \pm 7,5$ & $\operatorname{sim}$ \\
\hline $\mathrm{C}$ & 59,0 & $25,4 \pm 1,0$ & 60,5 & 47,0 & 42,3 & $49,9 \pm 9,4$ & $\operatorname{sim}$ \\
\hline D & 150,0 & 75 & 178 & 139 & 125 & $147 \pm 28$ & $\operatorname{sim}$ \\
\hline $\mathrm{E}$ & 170,0 & $116 \pm 1,6$ & 276,2 & 214,8 & 193,3 & $228,1 \pm 43,0$ & $\operatorname{sim}$ \\
\hline $\mathrm{F}$ & 170,0 & $111,8 \pm 12,4$ & 266,2 & 207,0 & 186,3 & $219,9 \pm 41,4$ & $\operatorname{sim}$ \\
\hline G & Isenta & $2,4 \pm 0,8$ & 5,7 & 4,4 & 4,0 & $4,7 \pm 0,9$ & $\operatorname{sim}$ \\
\hline $\mathrm{H}$ & isenta & $1,8 \pm 0,4$ & 4,3 & 3,3 & 3,0 & $3,5 \pm 0,7$ & $\operatorname{sim}$ \\
\hline
\end{tabular}

${ }^{a}$ Amostras de óleo mineral isolante (OMI), padronizadas empregando a cromatografia gasosa em $\mu \mathrm{g} \mathrm{mL}^{-1}$; ${ }^{\mathrm{b}}$ total de íons cloreto após a reação de desalogenação; ${ }^{~}$ conversão do total de íons cloretos para ascarel considerando os aroclor 1242 (42\% de cloro), 1254 (54\% de cloro) e 1260 (60\% de cloro); ${ }^{\mathrm{d}}$ média entre os três tipos de aroclor 1242, 1254 e 1260.

contaminação das amostras dentro da faixa de $\pm 50 \mu \mathrm{g} \mathrm{mL}^{-1}$, o que está em concordância com a norma técnica vigente, NBR 8371/9467. O procedimento proposto apresenta-se como uma alternativa simples e de baixo custo (kits similares importados apresentam custos elevados $)^{23}$ podendo ser empregado em análises de campo, pois todas as etapas propostas para o ensaio são de fácil operação.

\section{CONCLUSÕES}

Os resultados mostram que a desalogenação do ascarel em óleo mineral isolante via reação ARN é rápida e de fácil acompanhamento, o que a torna adequada para abertura de amostras organocloradas em metodologias, analíticas.

O procedimento potenciométrico, empregando eletrodo seletivo a cloreto, apresenta-se como uma proposta simples e de baixo custo, podendo ser empregada em análises de campo.

Todas as etapas propostas para o ensaio são de fácil operação, podendo ser realizadas por pessoas sem treinamento específico.

Os resultados demonstram que o procedimento proposto é adequado para monitoramento de faixas de contaminação de $\pm 50 \mu \mathrm{g} \mathrm{mL}^{-1} \mathrm{de}$ ascarel em óleos isolante de transformadores, o que está em concordância com a norma técnica vigente, NBR 8371/946.

Apesar de semi-quantitativo, o procedimento proposto caracteriza-se com uma solução econômica para o monitoramento da contaminação por ascarel em transformadores, uma vez que separa amostras com níveis acima e abaixo do permitido pela legislação, minimizando o número de análises a serem realizadas pela metodologia oficial.

\section{AGRADECIMENTOS}

Á CELESC - Centrais Elétricas de Santa Catarina.

\section{REFERÊNCIAS}

1. Penteado, J. C. P.; Vaz, J. M.; Quim. Nova 2001, 24, 390.

2. http://www.ambicare.com/pcb.htm, acessada em Dezembro 2006.

3. Salgado, P. E. de T.; Bifenilas Policloradas, Poluentes Orgânicos Persistentes, POPs, Série Cadernos de Referência Ambiental: Salvador, 2002, vol. 13, cap. 2.

4. Silva, J. A.; Tese de Mestrado, Universidade Estadual Paulista, Brasil, 1997.

5. Silva, L. R.; Ferreira, M. M.; Quim. Nova 2003, 26, 312.

6. Milasch, M.; Manutenção de Transformadores em Líquido Isolante, Edgard Blücher: São Paulo, 1984, cap. 12.

7. ABNT (Brasil), Ascarel para transformadores e capacitores - Características e riscos; NBR 8371/94, São Paulo, 1994.

8. International Electro-technical Commission, Insulating liquids Contamination by polychlorinated $(P C B s)$ - Method of determination by capillary column gas chromatography; IEC 61619/97, Switzerland, 1997.

9. Fieser, F. L.; Reagents for Organic Synthesis, John Wiley \& Sons Inc. 1967.

10. Wang, H. C.; Levin, G.; Szwarc, M.; J. Am. Chem.Soc. 1978, 100, 3969.

11. Stevenson, G. R.; Valentin, J.; Meverden C.; Echegoyen, L.; Maldonado, R.; J. Am. Chem.Soc. 1978, 100, 353.

12. Burke, S. D.; Danheiser, R. L.; Handbook of Reagents for Organic Synthesis, Oxidizing and Reducing Agents, John Willey \& Sons Ltd: New York, 2000.

13. APHA, AWWA \& WPCF Em Standard Methods for the Examination of Water and Wastewater; $14^{\text {th }}$ ed., Washington, (APHA 408c), 1975, p. 8745 .

14. Voguel, A.; Química Orgânica, $3^{\mathrm{a}}$ ed., Ao Livro Técnico: Rio de Janeiro, 1980, vol. 1.

15. Antonello, I.; Trabalho de Conclusão de Curso, Universidade Federal de Santa Catarina, Brasil, 2004.

16. Antonello I.; Dissertação de Mestrado, Universidade Federal de Santa Catarina, 2006.

17. Antonello, I.; Martins, A. R.; Souza, I. G.; Debacher, N. A.; Humeres, E.; Resumos do $13^{\circ}$ Encontro de Química Analítica e $1^{\circ}$ Congresso IberoAmericano de Química Analítica, Niterói, Brasil, 2005.

18. Kochi, J. K.; Free Radicals, Jonh Wiley \& Sons, Inc: New York, 1973, vol. 1 , cap. 9 .

19. McMurry, J.; Química Orgânica; 4ª ed., LTC: Rio de Janeiro, 1997, vol. 1 , cap. 16.

20. Smith, J. G.; Bubbar, G. L.; US pat. 4,326,090 1982

21. Parker, D. K.; Steichen, R. J.; US pat. 4,284,513 1981.

22. Brunelle, D. J.; US pat. 4,410,422 1983.

23. http://dexsil.com, acessada em Dezembro 2006.

24. EPA, (US); Screening test method for polychlorinated biphenyls in soil, Method 9078, 1996. 